\title{
S100B expression in breast cancer as a predictive marker for cancer metastasis
}

\author{
MENG-CHI YEN ${ }^{1,2}$, YUNG-CHI HUANG ${ }^{3}$, JUNG-YU KAN ${ }^{2,4}$, PO-LIN KUO ${ }^{2}$, \\ MING-FENG $\mathrm{HOU}^{2,4}$ and YA-LING HSU ${ }^{2,3}$ \\ ${ }^{1}$ Department of Emergency Medicine, Kaohsiung Medical University Hospital; ${ }^{2}$ Graduate Institute of \\ Clinical Medicine, and ${ }^{3}$ Graduate Institute of Medicine, College of Medicine, Kaohsiung Medical University; \\ ${ }^{4}$ Division of Breast Surgery, Kaohsiung Medical University Hospital, Kaohsiung 807, Taiwan, R.O.C.
}

Received September 12, 2017; Accepted December 5, 2017

DOI: $10.3892 /$ ijo.2017.4226

\begin{abstract}
In the tumor microenvironment, soluble molecules play important role in the establishment of a pre-metastatic niche. The S100 calcium-binding protein family are inflammatory molecules that contribute to the development of a pro-inflammatory tumor microenvironment. S100B belongs to the S100 family and serum S100B (also known as S100beta) serves as a marker for metastasis in lung cancer, ovarian cancer and melanoma. However, the association between S100B and the metastasis of breast cancer is not yet well understood. In the present study, a relatively low S100B expression was observed in the tumor samples compared to normal breast tissue among online microarray datasets. When the estrogen receptor (ER)-negative breast cancer cell lines, MDA-MB-231 and Hs578T, were treated with recombinant human S100B, cell migration was significantly inhibited and epithelial cadherin expression was increased. Our results revealed that a high S100B expression predicted a good overall survival in patients with ER-negative breast cancer, and good distant metastases-free survival in all patients with breast cancer via the analysis of the KM plotter and SurvExpress databases. Although previous studies have indicated that the interaction of S100B with wild-type p53 inhibits p53 function, a high S100B expression is associated with a good prognosis in patients with p53 mutant and p53 wild-type breast cancers. On the whole,
\end{abstract}

Correspondence to: Professor Ming-Feng Hou, Graduate Institute of Clinical Medicine, College of Medicine, Kaohsiung Medical University, 100 Shih-Chuan 1st Road, Kaohsiung 807, Taiwan, R.O.C.

E-mail: mifeho@kmu.edu.tw

Professor Ya-Ling Hsu, Graduate Institute of Medicine, College of Medicine, Kaohsiung Medical University, 100 Shih-Chuan 1st Road, Kaohsiung 807, Taiwan, R.O.C.

E-mail: hsuyl326@gmail.com

Key words: S100B, migration, epithelial-mesenchymal transition, p53 mutation, breast cancer our findings demonstrate that S100B treatment suppresses the migratory capacity of ER-negative breast cancer and that S100B expression may serve a predictive marker for metastasis in breast cancer.

\section{Introduction}

Breast cancer remains a leading cause of cancer-related mortality in women worldwide (1). Distant metastasis is a major cause of cancer-related mortality (2). Clinical studies have shown that breast cancer frequently metastasizes to the brain, lungs, bone, liver and lymph nodes (3). Compared to the cancer cells in the primary site, metastatic cells are less sensitive to breast cancer treatments (4). Therefore, the investigation of the factors which are involved in the development of a pre-metastatic niche is of utmost importance.

Inflammatory molecules, including chemokines, cytokines and other secreted molecules are a driving force that affects the microenvironment of extracellular matrix, and extracellular matrix remodeling is an important process for the establishment of a pre-metastatic niche (5). The S100 calcium-binding protein family are inflammatory molecules and contribute to develop an inflammatory tumor microenvironment (6). There are $>20$ genes in the S100 family and some of these are considered as tumor markers (7). A recent study suggested that S100A4 and S100P, which are members of the S100 family, serve as pro-metastatic factors (8). S100B (also known as S100beta) also belongs to the S100 family. A previous study also indicated that $\mathrm{S100B}$ overexpression led to the enhanced migratory and invasive capacity of a lung cancer cell line, promoting brain metastasis (9). Serum S100B and S100B autoantibodies are biomarkers of brain metastasis in lung cancer (10). In melanoma, the serum S100B concentration positively correlates with the tumor stage and negatively correlates with the survival rate $(11,12)$. Furthermore, elevated serum S100B levels are associated with the metastasis of melanoma, and decreased serum S100B levels are associated with an increased survival rate (13). In addition, S100B downregulates tumor suppressor p53 expression in melanoma. The knockdown of S100 expression restores p53-mediated apoptosis $(14,15)$. These data suggest that S100B is a molecule associated with tumor metastasis and progression. 
However, serum concentrations of S100B and HER2 may not be biomarkers of brain metastasis in breast cancer (16). By contrast, a recent study revealed that the serum levels of S100B were an independent prognostic determinant for patients with brain metastasis (17). In the serum of breast cancer patients subjected to endocrine therapy treatment, elevated levels of S100B are associated with a poor disease-free survival (18). Currently, the role of S100B in breast cancer is not well known and the association between the cell metastatic capacity and S100B has not yet been determined. Thus, in the present study, we aimed to investigate whether S100B directly regulates migration by performing in vitro experiments and using bioinformatics databases.

\section{Materials and methods}

Cells and cell culture. The human breast cancer cell lines, MCF-7 (ATCC ${ }^{\circledR}$ HTB-22 $^{\text {TM }}$ ), Hs578T (ATCC ${ }^{\circledR}$ HTB-126 ${ }^{\mathrm{TM}}$ ) and MDA-MB-231 (ATCC ${ }^{\circledast}$ HTB-26 ${ }^{\mathrm{TM}}$ ), were purchased from the American Type Culture Collection (ATCC, Manassas, VA, USA). The MCF-7, Hs578T and MDA-MB-231 cells were respectively cultured in Eagle's minimum Essential medium (EMEM), Dulbecco's modified Eagle's medium (DMEM) and L-15 medium supplemented with $10 \%$ fetal bovine serum (FBS) and 1\% penicillin-streptomycin (Thermo Fisher Scientific, Inc., Waltham, MA, USA). The MCF-7 and Hs578T cells were cultured at $37^{\circ} \mathrm{C}$ with $5 \% \mathrm{CO}_{2}$ and the MDA-MB-231 cells were cultured at $37^{\circ} \mathrm{C}$ in a $\mathrm{CO}_{2}$-free incubator.

Reverse transcription-quantitative PCR (RT-qPCR). Total RNA was isolated from the MCF-7, MDA-MB-231 and Hs578T cells using TRIzol reagent (Invitrogen, Carlsbad, CA, USA). The PrimeScript RT reagent kit (Clontech Laboratories, Inc., Kusatsu, Japan) was used for the reverse transcription of $500 \mathrm{ng}$ of total RNA to complementary DNA. The relative expression of S100B was determined using a Real-Time PCR system (StepOnePlus Real-Time PCT system; Applied Biosystems, Foster City, CA, USA) using Fast SYBR-Green Master Mix (Applied Biosystems) with the specific primer: S100B forward, 5'-AGGGAGACAAGCACAAGCTG-3' and reverse, 5'-CGTGGCAGGCAGTAGTAACC-3'. The expression of glyceraldehyde-3-phosphate dehydrogenase (GAPDH) was detected using the primers: forward, 5'-GAGTCAACG GATTTGGTCGT-3' and reverse, 5'-TTGATTTTGGAGGGA TCTCG-3'. Relative mRNA expression was normalized to the expression level of GAPDH and calculated using the $2^{-\Delta \Delta \mathrm{Ct}}$ method (19).

Cell migration assay. The migration ability was analyzed by wound healing assay and Transwell migration assay. For wound healing assay, $2.4 \times 10^{5}$ MDA-MB-231 cells were seeded into 24-well plates. At $24 \mathrm{~h}$ after seeding, a scratch was made using a $200-\mathrm{ml}$ pipette tip. After scratching, cell debris was removed by washing twice with phosphate-buffered saline (PBS) and the cells were then incubated in serum-free L-15 medium containing 0, 0.1 and $1 \mathrm{nM}$ recombinant human S100B protein (R\&D Systems, Minneapolis, MN, USA). Images were acquired at 0 and $12 \mathrm{~h}$ following treatment with S100B. Quantification was performed using AxioVs40 V4.8.2.0 microimaging software (MicroImaging $\mathrm{GmbH}$, Gottingen,
Germany). QCM ${ }^{\mathrm{TM}}$ 24-well Cell Migration assay and Invasion System uncoated $8-\mu \mathrm{m}$ pore size polycarbonate membranes (Millipore, Billerica, MA, USA) were used for Transwell migration assay according to the manufacturer's instructions. A total of $4 \times 10^{4}$ MDA-MB-231 or Hs578T cells were seeded in $300 \mathrm{ml}$ of serum-free medium in 24-well upper inserts and $500 \mathrm{ml}$ medium with $10 \%$ FBS was placed in the lower chamber. After $12 \mathrm{~h}$, the bottom surface of the membrane was fixed in $5 \%$ formaldehyde solution, followed by $0.4 \mathrm{~g} / \mathrm{l}$ crystal violet (Sigma-Aldrich, St. Louis, MO, USA) staining for $2 \mathrm{~h}$. The cells on the upper surface were removed using a cotton swab after washing the crystal violet-stained membrane. The bottom of the membrane was then visualized using an inverted light microscope (Carl Zeiss Primo Vert Microscope; Carl Zeiss, Munich, Germany) at x100 magnification. In total, 4 random fields of view were counted and the relative fold of migration in each group was compared to the $0 \mathrm{nM} \mathrm{S100B}$ treatment group.

Western blot analysis. The cells were lysed on ice for $30 \mathrm{~min}$ in radioimmunoprecipitation lysis buffer (RIPA) buffer (Millipore) which was supplemented with protease inhibitor cocktail (Millipore) at a 100:1 ratio. The total cell lysate was collected following centrifugation at $4^{\circ} \mathrm{C}, 12,000 \mathrm{x}$ f for $15 \mathrm{~min}$. The protein concentration was determined using a bicinchoninic acid (BCA) protein assay kit (Thermo Fisher Scientific, Rockford, IL, USA). Equal amounts of protein were loaded and separated by $10 \%$ sodium dodecyl sulfate polyacrylamide gel electrophoresis (SDS-PAGE). The proteins were then transferred onto polyvinylidene difluoride membranes (Millipore). After $1 \mathrm{~h}$ of blocking [5\% skim milk in Tris-buffered saline with Tween-20 (TBST) buffer], the membranes were incubated with the following primary antibodies: N-cadherin (1:1,000, cat. no. 2167184; Millipore), E-cadherin (1:1,000, cat. no. 610182; BD Biosciences, San Jose, CA, USA), vimentin (1:1,000, cat. no. MAB3400; Millipore) and GAPDH (1:5,000, cat. no. MAB374; Millipore) at $4^{\circ} \mathrm{C}$ overnight. After washing with TBST, the membranes were incubated with secondary antibodies, including peroxidase conjugated goat anti-rabbit IgG (1:5,000; cat. no. AP132P; Millipore) and peroxidase conjugated goat anti-mouse IgG (1:5,000; cat. no. AP124P; Millipore) for $1 \mathrm{~h}$ at room temperature. The results were acquired on Alpha Innotech FluorChem FC2 Imaging system (ProteinSimple; Bio-Techne, Minneapolis, MN, USA).

Evaluation of S100B expression from public microarray datasets. The mRNA expression of S100B in breast cancer samples was obtained from the Oncomine Research Edition (Thermo Fisher Scientific; http://www.oncomine.org, v4.5) which includes the TCGA breast dataset of Ma et al (20). The clinical data of TP53 mutation and the expression levels of S100B in the breast cancer samples were downloaded as z-scores from the cBioPortal (http://www.cbioportal.org, Breast cancer, TCGA, Cell 2015) (21). The expression of S100B in different subtypes (PAM50) of breast cancer was evaluated using the GOBO database (http://co.bmc.lu.se/gobo) (22).

Assessment of the patient survival rate. The distant metastasisfree survival (DMFS) analysis of the patients with breast cancer 
A

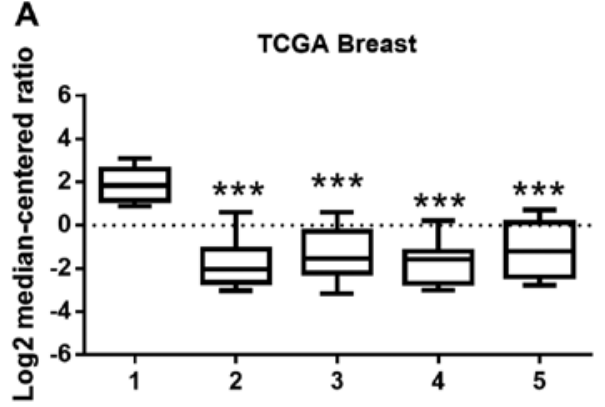

B

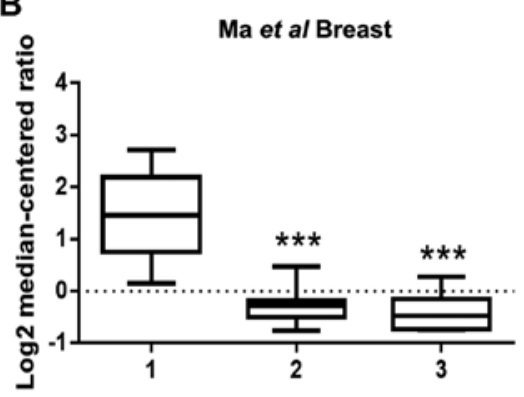

C

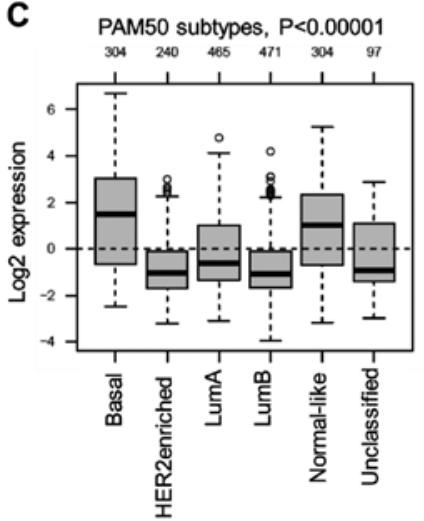

D

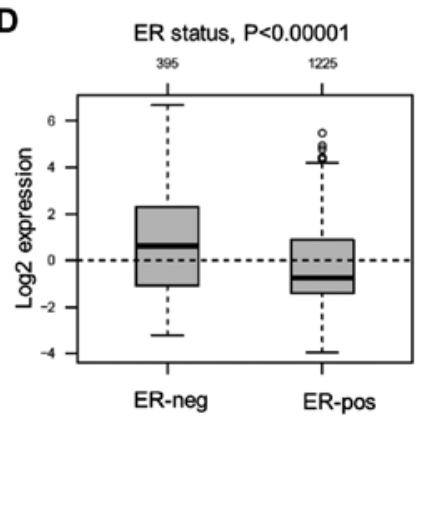

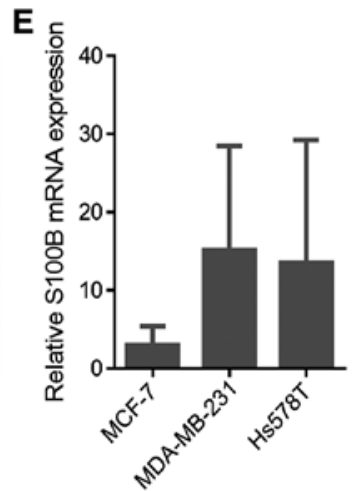

Figure 1. Expression levels of S100B in normal and tumor tissue. The expression of S100B was assessed from the Oncomine database. (A) TCGA breast dataset; the bars are labeled as follows: 1, breast (normal, $\mathrm{n}=61) ; 2$, invasive ductal breast carcinoma ( $=389$ ); 3 , invasive breast carcinoma ( $=76$ ); mixed lobular and ductal breast carcinoma $(\mathrm{n}=7)$; 5, invasive lobular breast carcinoma $(\mathrm{n}=36)$. (B) The Ma et al breast dataset (19); the bars are labeled as follows: 1 , breast carcinoma $(\mathrm{n}=14) ; 2$, ductal breast carcinoma in situ $(\mathrm{n}=9)$; 3, invasive ductal breast carcinoma $(\mathrm{n}=9)$. Boxplot shows the $\mathrm{z}$-score of S100B expression according to the raw data from Oncomine. Error bars represent standard deviation (SD). The statistically significant differences between each group were examined with one-way ANOVA ( $\left.{ }^{* * *} \mathrm{P}<0.001\right)$. (C) S100B expression in different types of breast cancer examined via the GOBO database. (D) S100B expression was examined in estrogen receptor (ER)-negative (ER-neg) and ER-positive (ER-pos) breast cancer using the GOBO database. The number above each bar indicates the sample size in each group. (E) mRNA expression of S100B in breast cancer cell lines. The error bars represent SD.

with different expression levels of S100B was performed using Kaplan-Meier plotter (KM Plotter) database (http://kmplot. com/analysis/) (23). Briefly, the prognostic value of each gene was analyzed by splitting the patient samples into 2 groups according to the median value. To investigate the association between S100B expression and DMFS in different subtypes of breast cancer patients, the DMFS was determined in the subtype of estrogen receptor $(E R)$-positive $(n=664)$ patients, ER-negative $(n=218)$ patients, or patients subjected to endocrine therapy $(n=645)$. The hazard ratio with $95 \%$ confidence intervals and log-rank P-value are shown according to the KM plotter database. To further examine whether the expression of S100B is associated with the TP53 status, the subtype of breast cancer was restricted to TP53 status 'mutated' $(n=188)$ or 'wild-type' $(n=273)$ and the DMFS of the patients was analyzed. The overall survival was accessed in the dataset 'Breast Invasive Carcinoma TCGA' from the SurvExpress database (http://bioinformatica.mty.itesm.mx:8080/Biomatec/ SurvivaX.jsp, Interface v2.0, Database Update: November 5, 2017) $(24,25)$. The censored was set to 'Survival_months', groups were divided by 'maximize risk groups' and stratification was set to 'class: ER'. To determine the distant recurrence rate, the dataset 'Vincent Darbon Breast' (Accession no. GSE9893 in the Gene Expression Omnibus database) in the SurvExpress database. The censored was set 'Distant recurrence months', and the raw data of the distant recurrence-free survival curve and mRNA expression levels were sequentially obtained.
The low- and high-risk groups were divided according to the median risk index on the SurvExpress website. The survival curve and mRNA expression were re-drawn using GraphPad Prism 7 software (GraphPad Software, Inc., San Diego, CA, USA).

Statistical analysis. All graphs were generated using GraphPad Prism 7 software (GraphPad Software). The Student's t-test was used for the analysis of differences between 2 groups and one-way analysis of variance (ANOVA) with a Tukey's post hoc test was used for the analysis of differences among 3 or more groups. A P-value $<0.05$ was considered to indicate a statistically significant difference.

\section{Results}

Relatively low $\mathrm{S100B}$ expression was observed in breast cancer. To investigate the expression levels of S100B in the breast cancer samples, the Oncomine database (http://www. oncomine.org) was used. Compared to the normal breast tissue, the expression of S100B in various types of breast cancer tissue was significantly low in 2 independent datasets (Fig. 1A and B). To further examine S100B expression in different subtypes of breast cancer according to PAM50 subtypes, S100B expression was evaluated using the GOBO database (http://co.bmc.lu.se/gobo). As shown in Fig. 1C, a relatively high $\mathrm{S100B}$ expression level was observed in the 


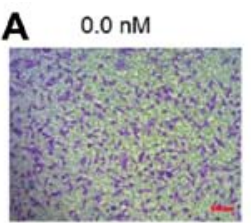

$1.0 \mathrm{nM}$

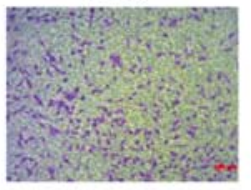

D

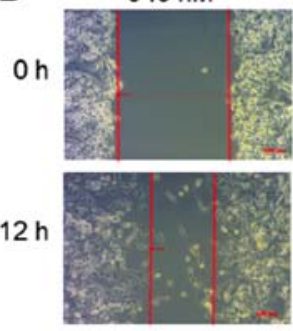

$0.1 \mathrm{nM}$

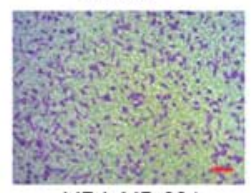

MDA-MB-231

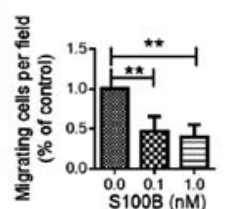

B $\quad 0.0 \mathrm{nM}$

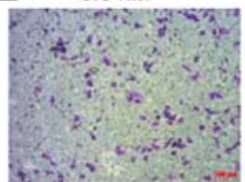

$1.0 \mathrm{nM}$

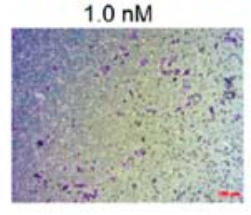

$10.0 \mathrm{nM}$
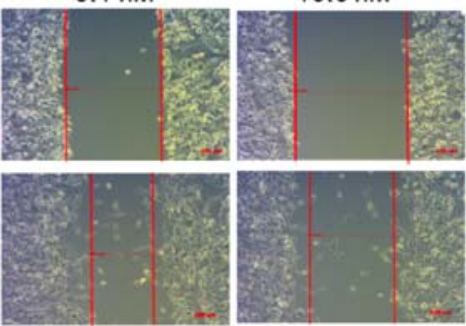

MDA-MB-231

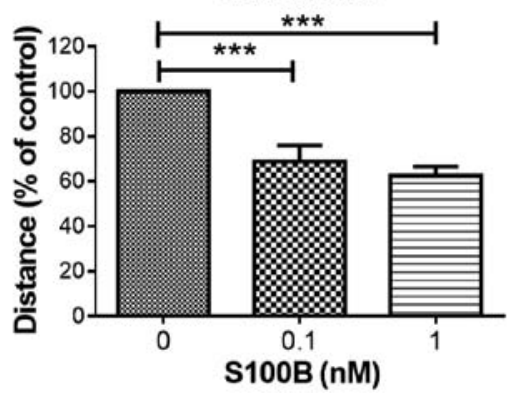

$0.1 \mathrm{nM}$

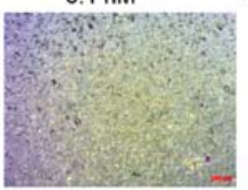

Hs578T

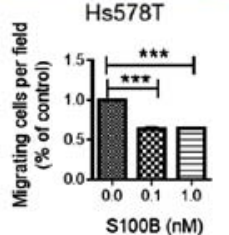

E

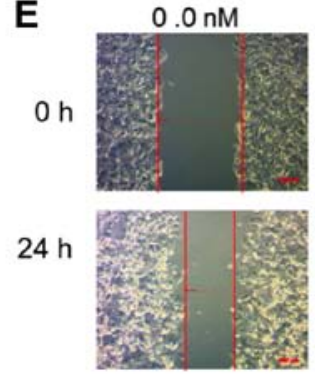

C $\quad 0.0 \mathrm{nM}$

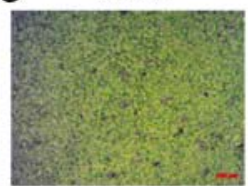

$1.0 \mathrm{nM}$

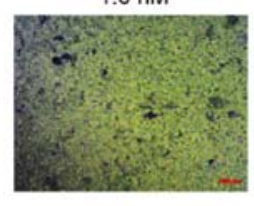

$0.1 \mathrm{nM}$

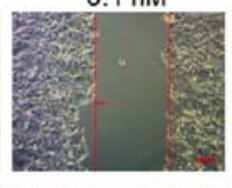

$0.1 \mathrm{nM}$

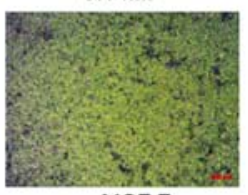

MCF-7

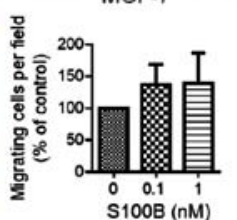

$1.0 \mathrm{nM}$

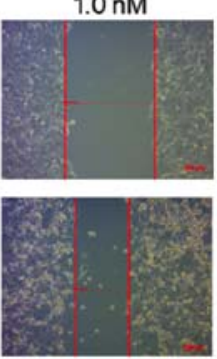

MCF-7

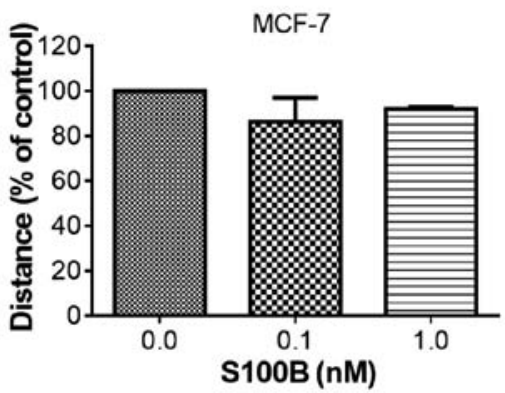

Figure 2. S100B treatment inhibits cell migration. Transwell migration assay in (A) MDA-MB-231 and (B) Hs578T and (C) MCF-7 cells. Images and quantification results are shown. Images of wound healing assay in (D) MDA-MB-231 and (E) MCF-7 cells. The quantification of wound healing assay in both cells is shown in the lower panel in the images. Error bars represent $\mathrm{SD}$ (one-way ANOVA; ${ }^{* *} \mathrm{P}<0.01$ and ${ }^{* * *} \mathrm{P}<0.001$ ).

basal-like type when compared to the luminal A (LumA), LumB and HER2-enriched groups. Moreover, S100B expression in the ER-negative group was higher than that in the ER-positive group (Fig. 1D). Based on these results, we hypothesized that S100B may directly regulate the behaviors of the breast cancer cells, particularly in basal-like or ER-negative breast cancer. mRNA expression levels in 3 cancer cell lines, including MCF-7 (luminal A, ER-positive), MDA-MB-231 (basal-like, ER-negative) and Hs578T (basal like, ER-negative) were then determined. As shown in Fig. 1E, the mean S100B expression in the MDA-MB-231 and Hs578T cells was higher than that in the MCF-7 cells; however, there was no statistical difference among these 3 cell lines.

Treatment with recombinant $S 100 B$ inhibits cell migration and promotes the epithelial-phenotype. The effects of extracellular S100B were further examined in the MCF-7, MDA-MB-231 and Hs578T cells. The cells were treated with various concentrations of human recombinant S100B protein. In the Transwell migration assay, S100B treatment significantly inhibited the migration of the two basal-like cell lines (Fig. 2A and B); however, it did not significantly affect the migration of the MCF-7 cells (Fig. 2C). In addition, the inhibitory effects of S100B treatment were observed in the
MDA-MB-231 cells (Fig. 2D), but not in the MCF-7 (Fig. 2E) cells, in wound healing assay. The process of epithelialmesenchymal transition (EMT) in cancer cells is associated with metastatic potential in breast cancer and EMT is related to the basal-like type of breast cancer (26). The MDA-MB-231 and Hs578T cells are classified as the basal B type (27). In order to determine whether S100B affects the mesenchymal or epithelial phenotype transition, the markers of the mesenchymal phenotype, $\mathrm{N}$-cadherin and vimentin, and the marker of the epithelial phenotype, E-cadherin (28), were determined in the MDA-MB-231 cells. The results of western blot analysis revealed that $\mathrm{S} 100 \mathrm{~B}$ treatment induced the expression of E-cadherin. Thus, S100B treatment led to the acquirement of the epithelial phenotype (Fig. 3).

High S100B expression is associated with a good prognosis. As S100B treatment inhibited cell metastasis and the acquisition of the epithelial phenotype, we further investigated whether S100B expression could be a prognostic marker for metastasis in patients with breast cancer, particularly in patients with metastatic breast cancer via the online databases, SurvExpress (http://bioinformatica.mty.itesm.mx:8080/ Biomatec/SurvivaX.jsp) and KM plotter (http://kmplot.com/ analysis/). In a dataset from the SurvExpress database, patients 
A

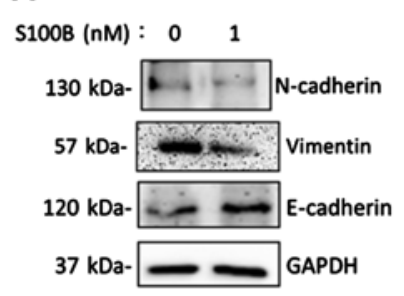

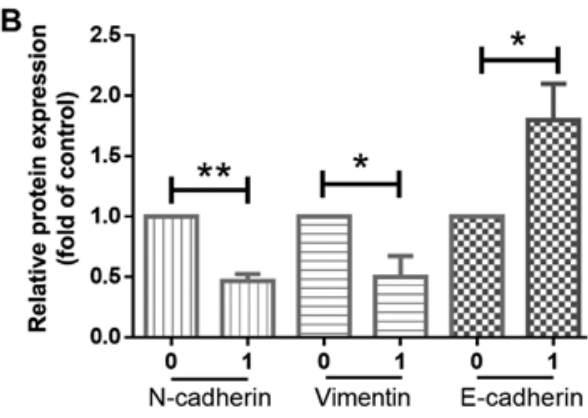

Figure 3. S100B treatment induces mesenchymal-epithelial transition (MET) in MDA-MB-231 cells. (A and B) Western blot analysis was used to examine the protein expression levels of MET signaling pathway-related molecules. Band intensity was normalized to glyceraldehyde 3-phosphate dehydrogenase (GAPDH). The relative value of the control $(0 \mathrm{nM})$ was set to 1 . Error bars represent $\mathrm{SD}$ (t-test; ${ }^{*} \mathrm{P}<0.05$ and $\left.{ }^{* * *} \mathrm{P}<0.01\right)$.

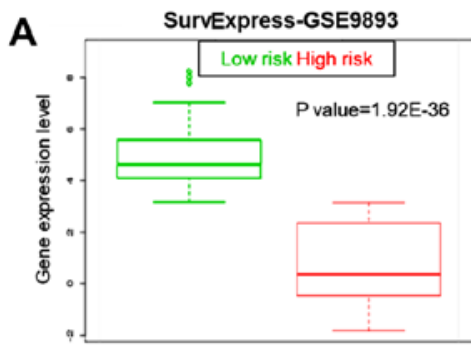

C

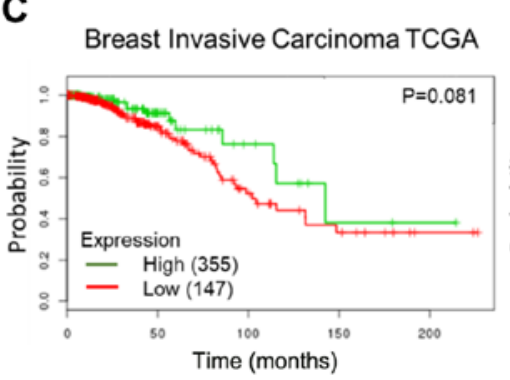

$\mathbf{F}$

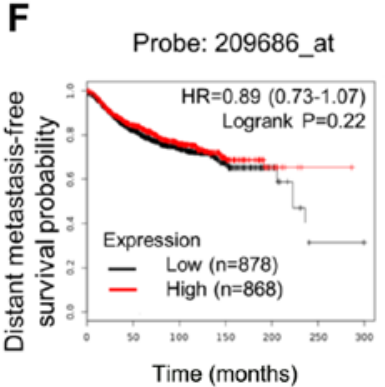

G

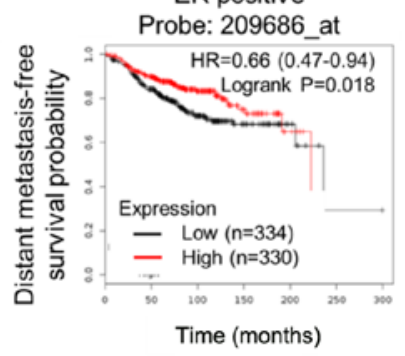

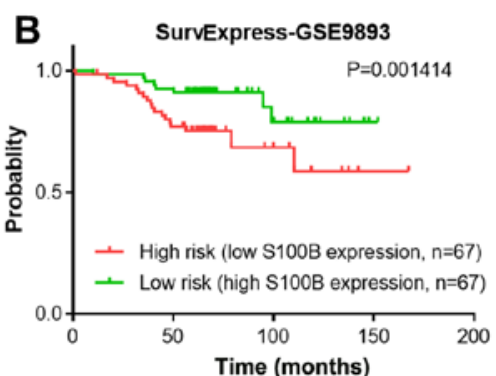

D Breast Invasive Carcinoma TCGA (ER-positive)
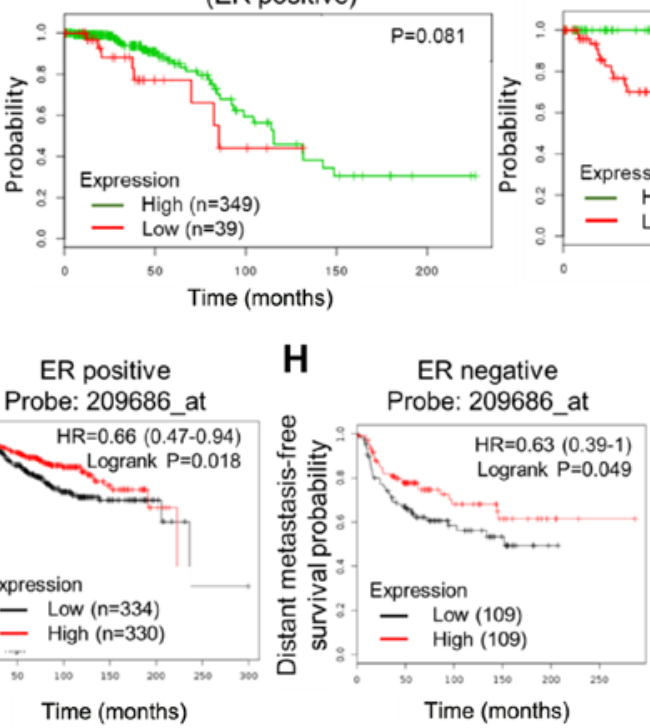

E Breast Invasive Carcinoma TCGA (ER-negative)

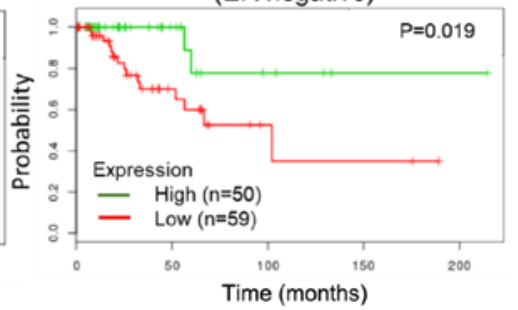

Figure 4. Evaluation of the association between S100B expression and survival curve. (A) S100B expression in the low-risk (green) and high-risk (red) group. The box plot is adapted from the SurvExpress database and shows S100B expression in patients. (B) Kaplan-Meier distant recurrence curve was created using the SurvExpress database to analyze the sample from a GEO dataset (GSE9893). The low-risk $(\mathrm{n}=67)$ and high-risk ( $\mathrm{n}=67)$ groups are shown in green and red, respectively. (C) Analyzing the overall survival rate in all breast cancer patients, (D) estrogen receptor (ER)-positive breast cancer patients, and (E) ER-negative breast cancer patients in 'breast invasive carcinoma TCGA' from the SurvExpress database. The green and red lines indicate a high and low S100B expression, respectively. Evaluation of the distant metastasis-free survival (DMFS) curve comparing the patients with a high (red) and low (black) S100B expression in (F) all breast cancer patients, (G) ER-positive breast cancer patients, (H) ER-negative breast cancer patients and (I) endocrine therapy-treated breast cancer patients using the KM plotter database. The hazard ratio (HR) and log-rank P-values are shown in each panel.

with a low S100B expression (high-risk group) had a good survival rate in a distant recurrence-free survival analysis (Fig. 4A and B). Furthermore, we examined whether the ER status was an important factor in the S100B-induced inhibitory effect on cell migration. In breast invasive carcinoma, the TCGA dataset from the SurvExpress database, a high S100B expression was associated with a good overall survival rate in only ER-negative breast cancer patients, but not in ER-positive breast cancer patients and in breast cancer patients as a whole (Fig. 4C-E). As S100B treatment resulted in the inhibition of the migration of ER-negative breast cancer cell lines, we further investigated the DMFS using the KM plotter database. S100B expression was not associated with DMFS in all breast cancer patients as a whole (Fig. 4F). By contrast, S100B 

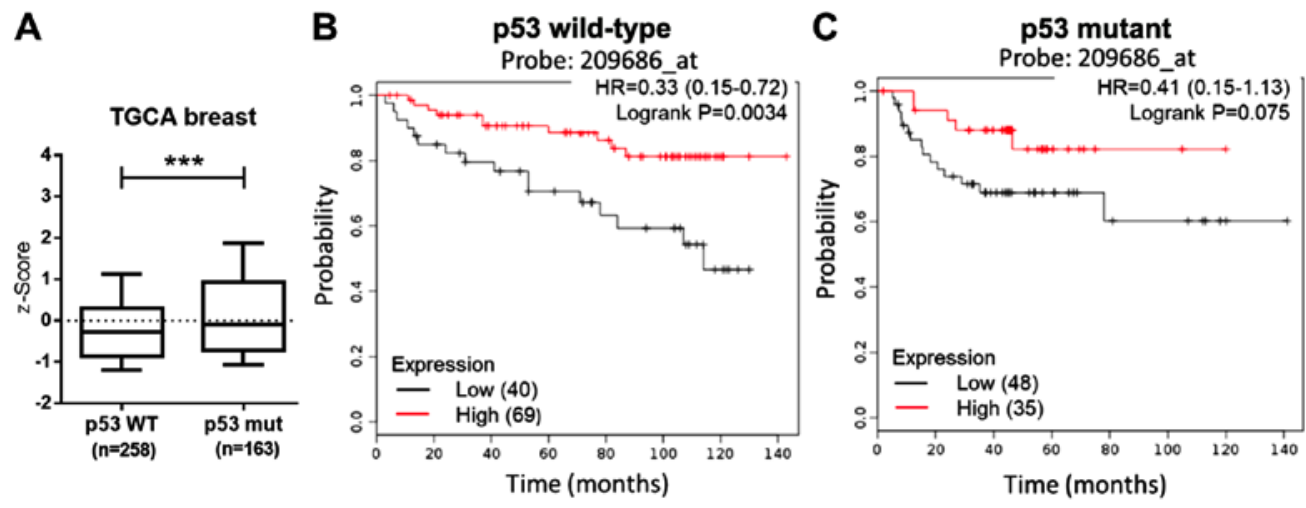

Figure 5. Evaluation of whether p53 expression in human breast cancer is associated with S100B expression and patient survival. (A) Expression of S100B in patients with or without p53 mutation. Raw data were adapted from the TCGA breast dataset. Distant metastasis-free survival (DMFS) analysis in breast cancer patients with (B) p53 mutation and (C) p53 wild-type. The hazard ratio (HR) and log-rank P-value are shown in each panel.
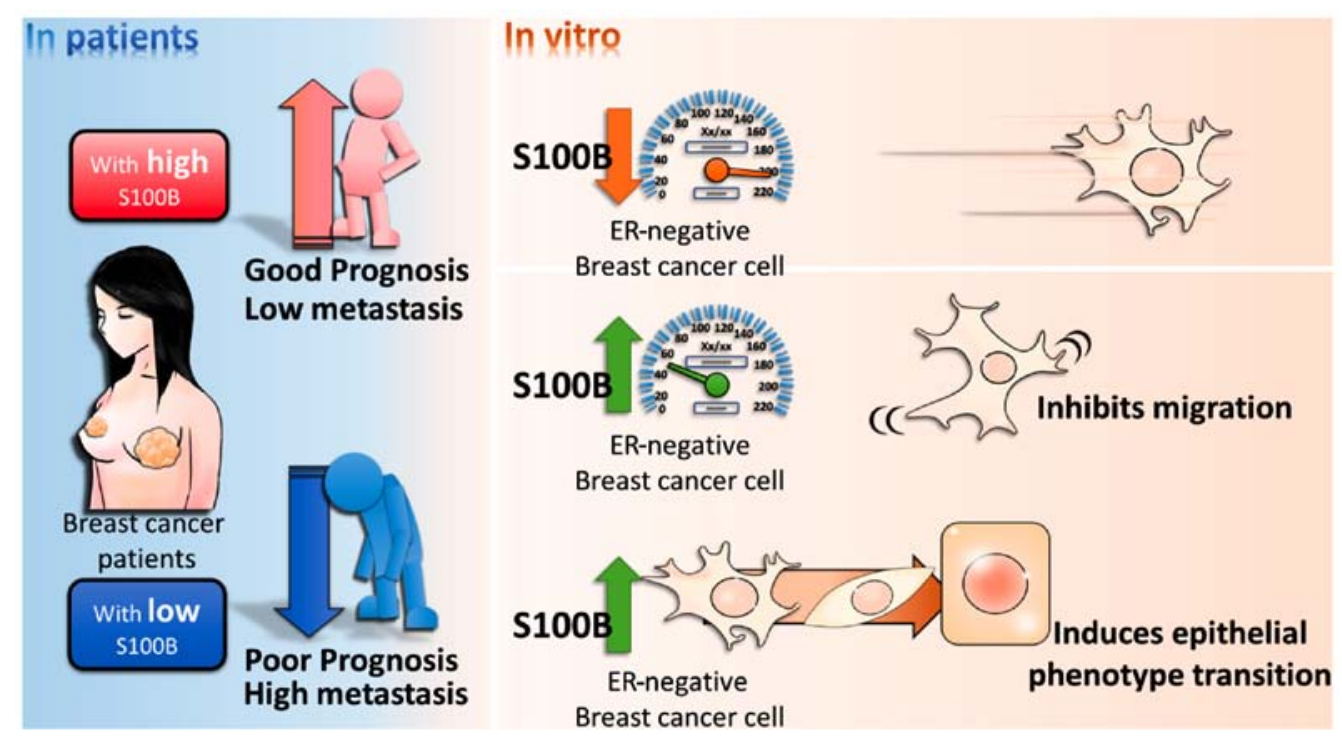

Figure 6. Summary of the S100B-mediated effects in breast cancer. Patients with breast cancer expressing high levels of S100B exhibited a good prognosis and a low metastatic rate. When the breast cancer cells are treated with $\mathrm{S100B}$, the migration ability was inhibited and the epithelial phenotype was induced.

expression was associated with a good DMFS in ER-positive $(\mathrm{P}=0.018)$ and ER-negative $(\mathrm{P}=0.049)$ breast cancer patients (Fig. 4G and H). Of note, S100B expression was a marker of prediction of DMFS in ER-positive breast cancer patients who received endocrine therapy (Fig. 4I).

High S100B expression is associated with good prognosis. In melanoma, S100B decreases p53 activity and expression $(14,15)$. In this study, the association between S100B and p53 expression was examined using the TCGA breast dataset from cBioPortal (http://www.cbioportal.org). Compared to the 553 wild-type group, a significantly higher p53 expression was observed in the p53 mutant group (Fig. 5A). Both the MDA-MB-231 and Hs578T cells exhibited p53 mutation (27). However, in this study, S100B treatment resulted in the inhibition of cell migration. Therefore, the association between the p53 status, S100B expression and the prognosis of breast cancer patients was evaluated. The DMFS in breast cancer patients with mutant p53 and wild-type p53 was respectively accessed in the KM plotter database. The results revealed that a high S100B expression was associated with a good outcome
(Fig. 5B and C). These findings suggest that a low S100B expression is associated with a high metastatic rate irrespective of the p53 status in breast cancer.

\section{Discussion}

Inflammation is a critical to create a pre-metastatic niche in cancer (5). The interaction of inflammatory molecules which are secreted from tumor cells, adipocytes and tumor-infiltrating macrophages, neutrophils, myeloid-derived suppressor cells and other types of immune cells regulate the behaviors of tumor cells (29). Metastasis-related pathways, such as the transition between the epithelial and mesenchymal phenotype, were altered by inflammatory molecules (29). Tumor cells secrete interleukin (IL)-6 and IL-8, which are important mediators of EMT in breast cancer (30). In addition, tumor cells secrete IL-6 and IL-8, which affect the phenotypes in an autocrine manner and IL-8 promotes the mesenchymal phenotype $(31,32)$. S100B is an inflammatory mediator and secreted from several types of cells, such as cancer cells and neuronal cells. Previous studies have suggested that S100B-expressing neuronal cells 
exert an autocrine/paracrine effect under physiological conditions $(32,33)$. We thus hypothesized that S100B may directly regulate the phenotype of breast cancer in an autocrine or paracrine manner. Therefore, this study focused on S100B expression in breast cancer cells and on the effects of S100B treatment. Of note, a previous study suggested that $\mathrm{S} 100 \mathrm{~B}$ was associated with brain metastasis in lung cancer (10). Thus, the tumor microenvironment may be a key factor which results in controversial effects in breast cancer. Compared to lung cancer, breast cancer grows near adipose tissue. Adipocytes secrete many molecules which regulate tumorigenesis and metastasis (34). In addition, adipocytes are an important source of serum S100B (35). The interaction of soluble factors and $\mathrm{S} 100 \mathrm{~B}$ in the tumor microenvironment exert differential effects on lung cancer cells and breast cancer cells.

There are $>20$ molecules in the S100 calcium-binding protein family. Different molecules have specific functions and regulatory mechanisms in different types of cancer. For example, S100A8 and S100A9 are associated with metastasis in breast cancer (36). By contrast, the overexpression of S100A6 inhibits metastasis and invasion in human osteosarcoma (37). Our results revealed a relatively high S100B expression in normal breast tissue, as observed by bioinformatics analysis when compared to breast cancer tissue. Furthermore, recombinant S100B treatment significantly suppressed cell migration and promoted the epithelial phenotype in ER-negative breast cancer cell lines. Our results thus suggest that S100B treatment can trigger the activation of anti-metastatic signaling pathways in ER-negative breast cancer. As the serum S100B level is not correlated with the number of metastatic sites of brain metastases in breast cancer (17), and S100B may affect the behavior of breast cancer cells in an autocrine or paracrine manner, we hypothesized that the expression S100B in breast cancer was not associated with the serum S100B levels. The detailed regulatory mechanisms of S100B expression warrant further investigation in breast cancer.

Our results revealed that $\mathrm{S} 100 \mathrm{~B}$ expression in ER-negative group was higher than that in the ER-positive group, as shown by bioinformatics analysis (Fig. 1D). RT-qPCR analysis revealed a similar trend in breast cancer cell lines (Fig. 1E). S100B expression was associated with a good overall survival rate in patients with ER-negative breast cancer, and a good DMFS in breast cancer patients as a whole. However, current endocrine therapy-treated patients with a high S100B expression and ER-positive breast cancer reveals poor disease-free survival (18). Based on these findings, it is suggested that ER signaling pathways may affect S100B expression and function. The anti-migratory effect of S100B was not observed in ER-positive MCF-7 cells. In microglia, extracellular S100B, IL-1 $\beta$, and tumor necrosis factor- $\alpha$ activate the NF- $\kappa$ B and AP-1 transcriptional activity and then results in the upregulation of cyclo-oxygenase 2 (38). The interaction between S100B-mediated signaling pathways and ER signaling pathways warrants further investigation in breast cancer.

Previous studies have demonstrated that S100B binds to the c-terminal of p53 protein, which is an important tumor suppressor gene $(14,15)$. The interaction of S100B and p53 results in the inhibition of p53-mediated growth suppression and apoptosis in melanoma, lung cancer and ovarian cancer $(10,14,39)$. In this study, S100B treatment inhibited the migration in of 2 p53-mutated breast cancer cells and S100B expression was associated with a good prognosis. Although we did not examine whether S100B binds to mutant p53 in the MDA-MB-231 and Hs578T cells, and did not assess the effects of S100B on breast cancer cell lines carrying wild-type p53, the current results revealed that $\mathrm{S} 100 \mathrm{~B}$ was associated with an anti-migratory effect. The interaction between p53 and S100B may play a minor role in breast cancer. The association between S100B, wild-type p53, mutant p53 and distant metastasis in breast cancer warrants further investigation.

In conclusion, this study demonstrated a novel role of $\mathrm{S} 100 \mathrm{~B}$ in the migration of breast cancer cells. S100B expression in breast cancer tissue was lower than that in normal breast tissue, and S100 exerted an inhibitory effect on cell migration and the mesenchymal phenotype. The summarized model is shown in Fig. 6. These results suggest that the regulatory mechanisms of action of S100B in breast cancer differ from those in melanoma, lung cancer and ovarian cancer, and that S100B expression may provide a predictive marker for metastasis in breast cancer.

\section{Acknowledgements}

The present study was supported by grants from the Ministry of Science and Technology (MOST 104-2314-B-037-053-MY4; MOST 105-2314-B-037-037-MY3; MOST 106-2314-B-037046) and the Kaohsiung Medical University Hospital Research Foundation (KMUHS10601; KMUH105-5M23).

\section{References}

1. Siegel RL, Miller KD and Jemal A: Cancer Statistics, 2017. CA Cancer J Clin 67: 7-30, 2017.

2. Scully OJ, Bay BH, Yip G and Yu Y: Breast cancer metastasis. Cancer Genomics Proteomics 9: 311-320, 2012.

3. Chiang AC and Massagué J: Molecular basis of metastasis. N Engl J Med 359: 2814-2823, 2008.

4. Vanharanta S and Massagué J: Origins of metastatic traits. Cancer Cell 24: 410-421, 2013.

5. Peinado H, Zhang H, Matei IR, Costa-Silva B, Hoshino A, Rodrigues G, Psaila B, Kaplan RN, Bromberg JF, Kang Y, et al: Pre-metastatic niches: Organ-specific homes for metastases. Nat Rev Cancer 17: 302-317, 2017.

6. Lukanidin E and Sleeman JP: Building the niche: The role of the S100 proteins in metastatic growth. Semin Cancer Biol 22: 216-225, 2012.

7. Salama I, Malone PS, Mihaimeed F and Jones JL: A review of the S100 proteins in cancer. Eur J Surg Oncol 34: 357-364, 2008.

8. Mei Y, Yang JP and Qian CN: For robust big data analyses: A collection of 150 important pro-metastatic genes. Chin J Cancer 36: 16, 2017.

9. Pang X, Min J, Liu L, Liu Y, Ma N and Zhang H: S100B protein as a possible participant in the brain metastasis of NSCLC. Med Oncol 29: 2626-2632, 2012.

10. Choi H, Puvenna V, Brennan C, Mahmoud S, Wang XF, Phillips M, Janigro D and Mazzone P: S100B and S100B autoantibody as biomarkers for early detection of brain metastases in lung cancer. Transl Lung Cancer Res 5: 413-419, 2016.

11. Bonfrer JM, Korse CM, Nieweg OE and Rankin EM: The luminescence immunoassay S-100: a sensitive test to measure circulating S-100B: its prognostic value in malignant melanoma. Br J Cancer 77: 2210-2214, 1998.

12. Heizmann CW: S100B protein in clinical diagnostics: Assay specificity. Clin Chem 50: 249-251, 2004.

13. Schlagenhauff B, Schittek B, Ellwanger U, Stroebel W, Blum A, Schwarz M, Rassner G and Garbe C: Significance of serum protein S100 levels in screening for melanoma metastasis: Does protein S100 enable early detection of melanoma recurrence? Melanoma Res 10: 451-459, 2000. 
14. Lin J, Yang Q, Wilder PT, Carrier F and Weber DJ: The calciumbinding protein S100B down-regulates p53 and apoptosis in malignant melanoma. J Biol Chem 285: 27487-27498, 2010.

15. Yoshimura $\mathrm{C}$, Miyafusa $\mathrm{T}$ and Tsumoto $\mathrm{K}$ : Identification of small-molecule inhibitors of the human S100B-p53 interaction and evaluation of their activity in human melanoma cells. Bioorg Med Chem 21: 1109-1115, 2013.

16. Bechmann T, Madsen JS, Brandslund I, Lund ED, Ormstrup T, Jakobsen EH, Jylling AM, Steffensen KD and Jakobsen A: Predicting brain metastases of breast cancer based on serum S100B and serum HER2. Oncol Lett 6: 1265-1270, 2013.

17. Darlix A, Lamy PJ, Lopez-Crapez E, Braccini AL, Firmin N, Romieu G, Thézenas S and Jacot W: Serum NSE, MMP-9 and HER2 extracellular domain are associated with brain metastases in metastatic breast cancer patients: Predictive biomarkers for brain metastases? Int J Cancer 139: 2299-2311, 2016.

18. Charmsaz S, Hughes É, Bane FT, Tibbitts P, Mcllroy M, Byrne C, Cocchiglia S, McBryan J, Hennessy BT, Dwyer RM, et al: S100 $\beta$ as a serum marker in endocrine resistant breast cancer. BMC Med 15: 79, 2017.

19. Livak KJ and Schmittgen TD: Analysis of relative gene expression data using real-time quantitative PCR and the 2(-Delta Delta C(T)) Method. Methods 25: 402-408, 2001.

20. Ma XJ, Dahiya S, Richardson E, Erlander M and Sgroi DC: Gene expression profiling of the tumor microenvironment during breast cancer progression. Breast Cancer Res 11: R7, 2009.

21. Ciriello G, Gatza ML, Beck AH, Wilkerson MD, Rhie SK Pastore A, Zhang H, McLellan M, Yau C, Kandoth C, et al, TCGA Research Network: Comprehensive molecular portraits of invasive lobular breast cancer. Cell 163: 506-519, 2015.

22. Ringnér M, Fredlund E, Häkkinen J, Borg Å and Staaf J: GOBO: Gene expression-based outcome for breast cancer online. PLoS One 6: e17911,2011.

23. Györffy B, Lanczky A, Eklund AC, Denkert C, Budczies J, Li Q and Szallasi Z: An online survival analysis tool to rapidly assess the effect of 22,277 genes on breast cancer prognosis using microarray data of 1,809 patients. Breast Cancer Res Treat 123: 725-731, 2010

24. Aguirre-Gamboa R, Gomez-Rueda H, Martínez-Ledesma E, Martínez-Torteya A, Chacolla-Huaringa R, RodriguezBarrientos A, Tamez-Peña JG and Treviño V: SurvExpress: An online biomarker validation tool and database for cancer gene expression data using survival analysis. PLoS One 8: e74250, 2013.

25. Chanrion M, Negre V, Fontaine H, Salvetat N, Bibeau F, Mac Grogan G, Mauriac L, Katsaros D, Molina F, Theillet C, et al: A gene expression signature that can predict the recurrence of tamoxifen-treated primary breast cancer. Clin Cancer Res 14 $1744-1752,2008$

26. Sarrió D, Rodriguez-Pinilla SM, Hardisson D, Cano A, MorenoBueno $G$ and Palacios J: Epithelial-mesenchymal transition in breast cancer relates to the basal-like phenotype. Cancer Res 68 : 989-997, 2008
27. Chavez KJ, Garimella SV and Lipkowitz S: Triple negative breast cancer cell lines: One tool in the search for better treatment of triple negative breast cancer. Breast Dis 32: 35-48, 2010.

28. Lamouille S, Xu J and Derynck R: Molecular mechanisms of epithelial-mesenchymal transition. Nat Rev Mol Cell Biol 15: 178-196, 2014.

29. Dominguez C, David JM and Palena C: Epithelial-mesenchymal transition and inflammation at the site of the primary tumor. Semin Cancer Biol 47: 177-184, 2017.

30. Demirkan B: The roles of epithelial-to-mesenchymal transition (EMT) and mesenchymal-to-epithelial transition (MET) in breast cancer bone metastasis: Potential targets for prevention and treatment. J Clin Med 2: 264-282, 2013.

31. Giri D, Ozen M and Ittmann M: Interleukin-6 is an autocrine growth factor in human prostate cancer. Am J Pathol 159: 2159-2165, 2001

32. Horiguchi K, Fujiwara K, Higuchi M, Yoshida S, Tsukada T, Ueharu H, Chen M, Hasegawa R, Takigami S, Ohsako S, et al: Expression of chemokine CXCL10 in dendritic-cell-like S100 $\beta$ positive cells in rat anterior pituitary gland. Cell Tissue Res 357: 757-765, 2014.

33. Villarreal A, Seoane R, González Torres A, Rosciszewski G, Angelo MF, Rossi A, Barker PA and Ramos AJ: S100B protein activates a RAGE-dependent autocrine loop in astrocytes: Implications for its role in the propagation of reactive gliosis. J Neurochem 131: 190-205, 2014.

34. Nieman KM, Romero IL, Van Houten B and Lengyel E: Adipose tissue and adipocytes support tumorigenesis and metastasis. Biochim Biophys Acta 1831: 1533-1541, 2013

35. Gonçalves CA, Leite MC and Guerra MC: Adipocytes as an important source of serum S100B and possible roles of this protein in adipose tissue. Cardiovasc Psychiatry Neurol 2010: 790431, 2010

36. Lim SY, Yuzhalin AE, Gordon-Weeks AN and Muschel RJ: Tumor-infiltrating monocytes/macrophages promote tumor invasion and migration by upregulating S100A8 and S100A9 expression in cancer cells. Oncogene 35: 5735-5745, 2016.

37. Luo X, Sharff KA, Chen J, He TC and Luu HH: S100A6 expression and function in human osteosarcoma. Clin Orthop Relat Res 466: 2060-2070, 2008

38. Bianchi R, Giambanco I and Donato R: S100B/RAGE-dependent activation of microglia via NF-kappaB and AP-1 Co-regulation of COX-2 expression by S100B, IL-1beta and TNF-alpha. Neurobiol Aging 31: 665-677, 2010.

39. Yang T, Cheng J, Yang Y, Qi W, Zhao Y, Long H, Xie R and Zhu B: S100B mediates stemness of ovarian cancer stem-like cells through inhibiting p53. Stem Cells 35: 325-336, 2017. 